\title{
Capability of a neck worn device to measure sleep/wake, airway position, and differentiate benign snoring from obstructive sleep apnea
}

\author{
Daniel J. Levendowski • Bratislav Veljkovic • \\ Sean Seagraves • Philip R. Westbrook
}

Received: 24 September 2013/ Accepted: 26 February 2014/Published online: 6 March 2014

(C) The Author(s) 2014. This article is published with open access at Springerlink.com

\begin{abstract}
To evaluate the accuracy of a neck-worn device in measuring sleep/wake, detecting supine airway position, and using loud snoring to screen for obstructive sleep apnea. Study A included 20 subjects who wore the neckdevice during polysomnography (PSG), with 31 records obtained from diagnostic and split-night studies. Study B included 24 community-based snorers studied in-home for up to three-nights with obstructive sleep apnea (OSA) severity measured with a validated Level III recorder. The accuracy of neck actigraphy-based sleep/wake was measured by assessing sleep efficiency (SE). Differences in sleep position measured at the chest and neck during PSG were compared to video-editing. Loud snoring acquired with an acoustic microphone was compared to the apneahypopnea index (AHI) by- and acrosspositions. Overreported SE by neck actigraphy was inversely related to OSA severity. Measurement of neck and chest supine position were highly correlated with video-edits $(\mathrm{r}=0.93$, 0.78). Chest was bias toward over-estimating supine time while the majority of neck-device supine position errors occurred during CPAP titrations. Snoring was highly correlated with the overall, supine, and non-supine PSG-AHI $(\mathrm{r}=0.79,0.74,0.83)$ and was both sensitive and specific in detecting overall, supine, and non-supine PSGAHI $>10$ (sensitivity $=81,88,82 \%$; specificity $=87,79,100 \%$ ). At home sleep testing-AHI $>10$, the sensitivity and
\end{abstract}

D. J. Levendowski $(\bowtie) \cdot$ B. Veljkovic · S. Seagraves ·

P. R. Westbrook

Advanced Brain Monitoring Inc., Carlsbad, CA, USA

e-mail:dan@b-alert.com

P. R. Westbrook

Professor Emeritus, School of Medicine, University of California

in Los Angeles (UCLA), Los Angeles, CA, USA specificity of loud snoring was superior when users were predominantly non-supine as compared to baseline (sensitivity $=100,92 \%$; specificity $=88,77 \%$ ). Neck actigraphy appears capable of estimating sleep/wake. The accuracy of supine airway detection with the neck-device warrants further investigation. Measurement of loud snoring appears to provide a screening tool for differentiating positional apneic and benign snorers.

Keywords OSA · Positional · Snoring · Screening · Actigraphy $\cdot$ Validation

\section{Introduction}

It has been estimated that the worldwide prevalence of snoring ranges from 30 to $50 \%$ of the adult population [1-9]. The odds of being a habitual snorer are greater in men $[1,3,8,9]$, with the prevalence further increased by age $[1,3,7,10]$, weight $[1,5,6]$, alcohol consumption $[1$, 5], and/or when pregnant [11-14]. Snoring is an independent risk factor for obstructive sleep apnea (OSA) [15, 16]. Approximately $50 \%$ of those who snore are likely to have OSA [17-20], and a substantial majority is undiagnosed [20]. The vibrations caused by heavy snoring have also been suggested as an independent risk factor for carotid atherosclerosis [21-24]. Of the $15-25 \%$ of the adult population who are benign snorers [i.e., apneahypopnea index $(\mathrm{AHI})<5$ ], most snore predominantly in the supine position $[25,26]$.

Recognition of the clinical importance of OSA risk factors and treating OSA is increasing worldwide, however an inexpensive, objective tool to differentiate those in need of a diagnostic sleep study from those with just benign snoring remains elusive. Such a tool might also be useful 
for large epidemiological assessments of snoring and OSA prevalence, the impact of snoring on cardiovascular disease, or the changes in snoring during pregnancy.

Previous reports suggest that the measurement of snoring with an acoustic microphone holds potential for noninvasively estimating sleep disordered breathing severity [27-31]. Fiz et al. [28] found that as AHI severity increases, the acoustics change, suggesting the maximum snoring frequency is reduced, the mean snoring intensities increase, and the ratio between the 100 and $500 \mathrm{~Hz}$ power expand. With respect to identifying excess tissue associated with airway collapse, fast-Fourier transform of high frequency snoring showed that palatal snoring lies in the lower frequencies of the sound spectrum with snoring frequencies increase stepwise from pharyngeal lateral wall, tongue based, and epiglottis obstruction [32]. Mikami et al. [30] showed that the fundamental frequency and maximum amplitudes in frequency spectrum can be used to distinguish oral snoring sounds in patients with and without sleep apnea. Fiz et al. [28] reported that receiver operating curves using acoustic snoring could provide sensitivities and specificities of 98.0 and 71.4 for an AHI $\geq 5$ and 80.0 and 90.0 for $\mathrm{AHI} \geq 15$ so long as sleeping position was factored into the snoring parameters. Abeyratne et al. [33] validated a OSA screening tool that used a multi-feature class analysis of snoring sounds acquired during laboratory polysomnography that provided sensitivities and specificities of $93 \%$ with an AHI cut-off of 15 events/h. Ben-Israel et al. [27] achieved a sensitivity of 0.87 and a specificity 0.80 with a non-contact microphone placed 1 meter above the bed at a sleep center.

Measuring the influence of position on snoring and/or OSA is important given $65 \%$ of benign snorers are positional and over $70 \%$ of patients with mild to moderate OSA are twice as severe supine as compared to non-supine [25]. Assessing snoring patterns relative to position may prove useful in monitoring outcomes with position restriction treatment, as well as other OSA therapies.

This report provides a description of a neck-worn device and a retrospective assessment of its capability to accurately measure sleep position and sleep/wake, and differentiate benign snorers from those with clinically relevant OSA.

\section{Methods}

\subsection{Description of neck-device}

The battery-powered, neck-worn device (Night Shift ${ }^{\mathrm{TM}}$, Advanced Brain Monitoring, Carlsbad, CA, USA) weighs $44 \mathrm{~g}$ and includes electronics housed in a 5.5 (1) $\times 3.8$ $(\mathrm{w}) \times 1.6(\mathrm{~h}) \mathrm{cm}$ enclosure affixed on the back of the neck

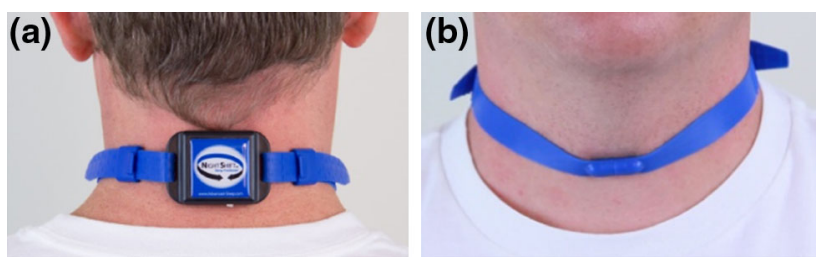

Fig. 1 Photograph of neck-device from $\mathbf{a}$ back and $\mathbf{b}$ front

with an adjustable non-latex silicone rubber strap secured by a magnetic clasp (see Fig. 1).

The neck-device measures snoring with a built-in acoustic microphone (PUI audio POM-2246P). The raw audio input is sampled at $2 \mathrm{kHz}$, and root mean square (RMS) of the digitized signal (12-bit A/D) is calculated using a $100 \mathrm{~ms}$ window. The resulting $10 \mathrm{~Hz}$ RMS signal is additionally filtered with a $0.5 \mathrm{~Hz}$ LP Butterworth 4th order filter. A snore algorithm quantifies each snore based on shape (attack, plateau and decline) and the peak amplitude, prior to conversion to $\mathrm{dB}$. Decibel calibration was performed using a sound level meter and recorded snoring sounds at $12 \mathrm{~cm}$. Snores $>40$ and $50 \mathrm{~dB}$ are tallied across each 30 -s epoch. Loud snoring is defined as at least one snore with a magnitude $\geq 50 \mathrm{~dB}$.

The percentage of time snoring $>50 \mathrm{~dB}$ is then determined for overall, supine, and non-supine epochs characterized as sleep by actigraphy. Snoring indicative of clinically relevant sleep disordered breathing is based on $>50 \mathrm{~dB}$ snoring in $\geq 10 \%$ of neck-based sleep time.

A three-axis accelerometer is used to measure neck position and actigraphic sleep versus wake. Neck positions are reported as upright, supine, lateral left, lateral right, and prone. Upright is assigned when the neck angle is $\geq 60^{\circ}$. Supine is assigned when the neck angle to the left/right is $<43^{\circ}$. Lateral left or right was assigned when the neck angle exceeded $47^{\circ}$. The device remained in the previously assigned position when the neck angle fell between $43^{\circ}$ and $47^{\circ}$. Prone is the mirrored position of supine with the additional requirement that the $\mathrm{Z}$ axis is $<-15^{\circ}$. If the device is worn upside down, the supine position will be accurately measured however lateral left and right will be inversed.

Sleep versus wake is measured in 30 -s epochs using a threshold applied to the median filter output derived from the three $\mathrm{X}, \mathrm{Y}$, and $\mathrm{Z}$ signals. If any of the three signals have an angle $<50^{\circ}$ and exceeds the actigraphy threshold, the epoch is classified awake. Periods with gross movement extend the wake classification for up to 3 epochs. The initial 10-min after the device is turned on is automatically classified as wake.

Two $\times 1 \mathrm{G}$ haptic motors optionally provide vibro-tactile feedback when the supine position is detected. 
Positional feedback, initiated at a very low frequency/ duration, is gradually increased until the user exits the supine position. When used for positional therapy, vibrotactile feedback is defaulted to initiate 15 -min after the device is turned on, to allow the user time to fall asleep. The user can optionally delay feedback for 30-min or set the device for immediate feedback. The device can also be used only as a recorder with positional feedback disabled.

Data are acquired and analyzed in real time with derived measures for sleep/wake, snoring magnitude, and position for each 30-s epoch saved to the microcontroller flash memory. The memory can store up to six nights' of detailed snoring, sleep, and position measures (one set of values for each 30-s epoch), summary of key daily parameters by month for 4 months, and the average values across the days in the month for 12 months. This information is accessed via assessment/compliance reports generated in HTML format from a web-enabled portal. The neck-device can record and provide vibro-tactile feedback for three nights before charging is required.

For this study, prototype devices were used that allow raw data to be saved to a memory card for off-line analysis using software which allows the records to be synchronized.

\subsection{Study A}

Consecutive volunteers scheduled for laboratory polysomnography (Complete Sleep Solutions, Murrieta, CA, USA) between the ages of 18 and 75 with a body mass index (BMI) $<35$ were recruited to wear the neck-device. Twenty subjects were consented under a protocol approved by the Chesapeake Institutional Review Board (IRB). This cohort included fifteen males and five females with a mean age of $46 \pm 13.2$ years and a mean BMI of $29 \pm 4.1 \mathrm{~kg} / \mathrm{M}^{2}$.

For this study, the neck-device was set to record mode (i.e., no positional feedback). The device was applied and turned on by the technician. Patients were instructed to sit upright in bed for 1 min just prior to lights out and just after lights on, to provide a means to synchronize the polysomnography (PSG) and the neck-device records.

The sleep studies were conducted and scored according to the American Academy of Sleep Medicine (AASM) criteria with $3 \%$ hypopnea desaturation [34]. Alice three or five systems were used with Pro-Tech nasal pressure transducers and Pro-Tech respiratory induced plethysmography effort sensors (Philips Respironics, Monroeville, PA, USA), and either Pro-Tech or SleepMate (Ambu, Inc. Glen Burke, MD, USA) actigraphy-based body (chest) position sensors.

\subsection{Study B}

Twenty-five individuals responding to a newspaper advertisement met the inclusion criteria of: (a) age between 30 and 85, (b) slept nightly with a bed partner who complained about their snoring, and (c) were not currently being treated with CPAP for OSA. Subjects were consented with a protocol approved by the BioMed IRB. This cohort included 14 males and 10 females with a mean age of $44 \pm 10.5$ years and a mean BMI of $31 \pm 7.7 \mathrm{~kg} / \mathrm{M}^{2}$ (range 22-57). Eighty-four percent were identified as being at high OSA risk by the STOP questionnaire [35] or by the ARES questionnaire [36, 37], with conflicting results in two cases. A prior diagnosis of OSA, high blood pressure and/or depression was reported in 12, 12 and $23 \%$ of the cases, respectively.

Subjects were instructed to wear the neck-device concurrent with a multi-channel home sleep testing (HST) device (ARES ${ }^{\mathrm{TM}}$, SleepMed, Boca Raton, FL, USA) for three nights. Night 1 was intended to provide a baseline, so the neck-device was set to record mode. On nights 2 and 3, the neck-device was set to feedback mode using preliminary supine detection parameters which under-reported supine sleep (e.g., neck angle $<35^{\circ}$ vs. $<43^{\circ}$ ).

The HST was affixed to the forehead and measured airflow from a cannula and pressure transducer, pulse and oximetry using reflective plethysmography, quantified snoring $(\mathrm{dB})$ with a calibrated acoustic microphone, and head movement and head position by actigraphy. Automated scoring algorithms were applied off-line to compute the AHI. Apneas, based on a 10-sec cessation of airflow detected by the automated algorithms, were included in the apnea-index (AI) and the AHI. Hypopnea events required a $50 \%$ reduction and recovery in airflow, and a minimum $3.5 \%$ reduction in $\mathrm{SpO}_{2}$ and at least a $1.0 \%$ recovery. Sleep time was determined behaviorally, based on actigraphy, snoring, and airflow patterns. The algorithms and accuracy of the automated scoring used to calculate the AHI have been previously reported [38-40].

Just prior to lights out, subjects were instructed to turn both devices on and perform a slow, loud 10-count, captured by both acoustic microphones and used to synchronize the records. Each morning subjects completed brief surveys to assess their sleep quality.

\subsection{Data analysis}

\subsubsection{Study A}

The Alice software was used to export: (a) EDF files to obtain epoch level resolution of chest position, (b) sleep 
stages for each epoch, (c) recording and sleep time, and (d) apnea/hypopnea indexes by, and across, positions. Technician notes from the video review were used to edit the body position.

To compare PSG and neck-device epochs classifications of sleep or wake and supine or non-supine, the clock times from the files were aligned, with accurate synchronization confirmed by the change to the upright position at the start of the recording. Comparisons of sleep versus wake were measured from lights out until lights on. Recording time was used to compare percentage of supine time from the chest and neck. Video recordings were reviewed to provide the gold-standard for classification of the supine position for both the chest and neck.

The overall percent time snoring $\geq 50 \mathrm{~dB}$ from the neck (loud snoring) was computed during periods detected as sleep by the neck actigraphy. From these sleep epochs, the supine and non-supine percent times snoring $\geq 50 \mathrm{~dB}$ were derived. Reporting of the percentage of positional time snoring required a minimum of 12-min of neck actigraphybased sleep time.

For the 11 participants who underwent a split-night study, snoring and AHI values were derived separately for the diagnostic and the CPAP titration periods. "Appendix 1" provides line data for the 20 subjects and 31 records used for analyses.

\subsubsection{Study B}

Prior to off-line analysis, both the HST and neck-device files were opened to identify the segment of the ARES EDF file which needed to be excluded so that the 10-counts were aligned.

\subsection{Statistical analyses}

Pearson correlations and Bland-Altman plots were used to characterize the association and differences between the paired measures. Two-tailed $t$ tests, which assumed equal variances, were used to measure significant between-measure differences. Cross-tabulations were used to compute the sensitivity, specificity, positive predictive value (PPV) and negative predictive value (NPV) between the overall, supine, and non-supine AHI and when loud snoring (i.e., $\geq 50 \mathrm{~dB}$ ) exceeded $10 \%$ of sleep time. Repeated measures analysis of variance (ANOVA) was used to assess significant changes in percent time supine across nights. Subjects identified with positional obstructive sleep apnea (POSA) required an overall $\mathrm{AHI} \geq 5$ and a supine/non-supine AHI ratio $\geq 2.0$ (based on $>12$-min of positional sleep time).

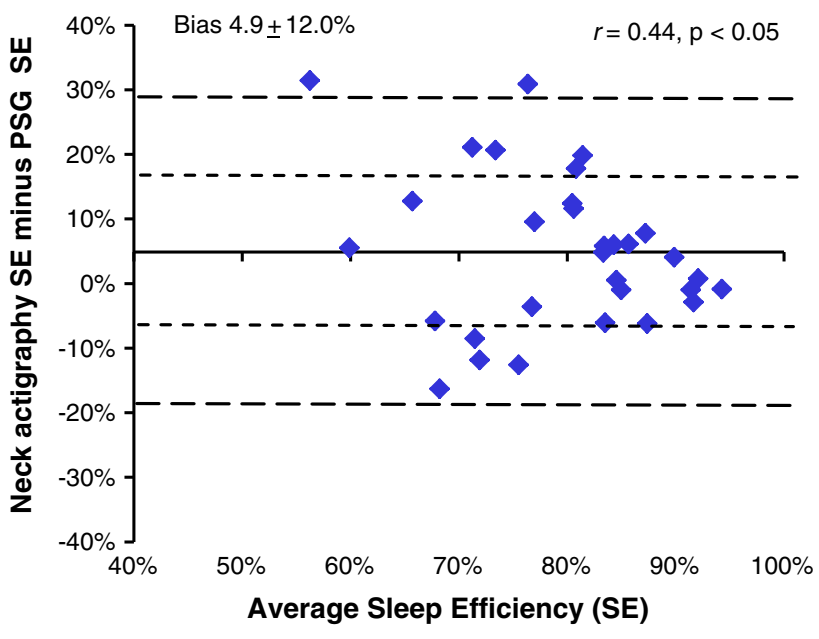

Fig. 2 Bland-Altman plot comparing sleep efficiency between PSG and neck actigraphy

\section{Results}

\subsection{Study A}

The mean $( \pm \mathrm{SD})$ sleep efficiency (SE) based on electrophysiology and actigraphy were $76.8 \pm 12.3$ and $81.8 \pm 10.0 \%$, respectively. No significant differences in SE were observed between PSG and the neck-actigraphy during the diagnostic studies $(73.8 \pm 12.1$ and $78.4 \pm 10.9$, respectively) or during the CPAP trials $(82.4 \pm 11.1$ and $87.9 \pm 3.7 \%$ ). Moderate agreement was observed between the PSG and neck actigraphy SE $(r=0.44)$. Neck actigraphy bias toward over-reporting sleep time (Fig. 2.) decreased as OSA severity increased. Biases of $9.9 \%$ observed for those with no OSA (AHI $<5$ ), $5.6 \%$ for those with mild to moderate OSA (AHI 5-29) and -4.9 for those with severe OSA $(\mathrm{AHI} \geq 30$ ).

Very strong, significant agreements were observed between the video-edited supine position and those measured by chest and neck ( $r=0.78$ and 0.93 respectively) (Fig. 3). When Subject 12 was removed, the correlation between chest and video-edited chest increased from 0.78 to 0.88 . The chest transducer over-reported supine time by $>5 \%$ in six studies, with an average error of $80 \mathrm{~min}$ (range 16-284). The average error in supine time decreased to 39 min when subject 12 was removed. In six cases, the absolute difference between supine time by neck position and video exceeded $5 \%$. In four cases, supine sleep time was over-reported by neck position by an average of 28 min (range 16-60 min), and in two cases, neck position under-reported supine sleep time by 18 and $38 \mathrm{~min}$. In only two cases, the neck and chest were reported in conflicting positions by video editing (Fig. 4). Subject 18 spent an 
(a)

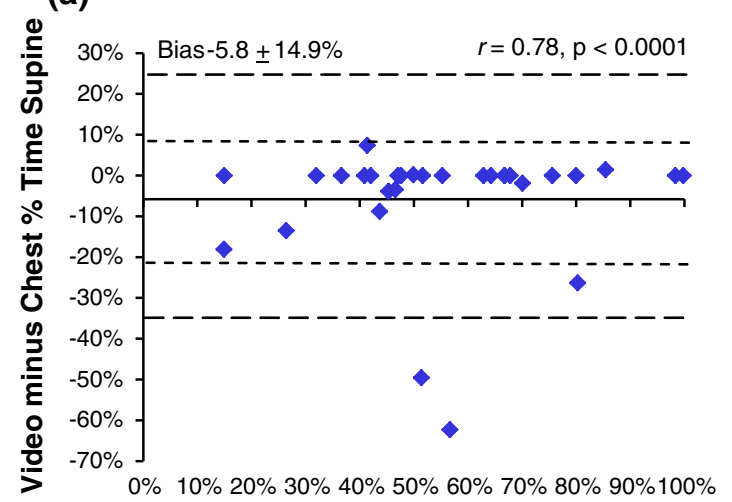

Average \% Time Supine (b)

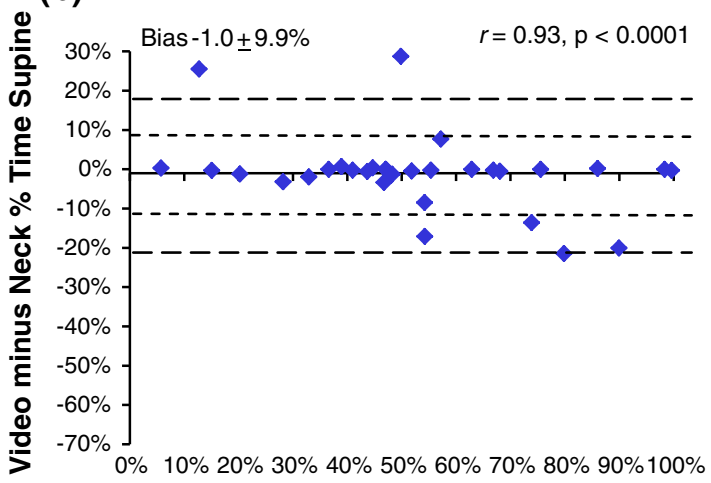

Average \% Time Supine

Fig. 3 Bland-Altman plots between edited and unedited actigraphy-based position from lights out to lights on from a chest and $\mathbf{b}$ neck
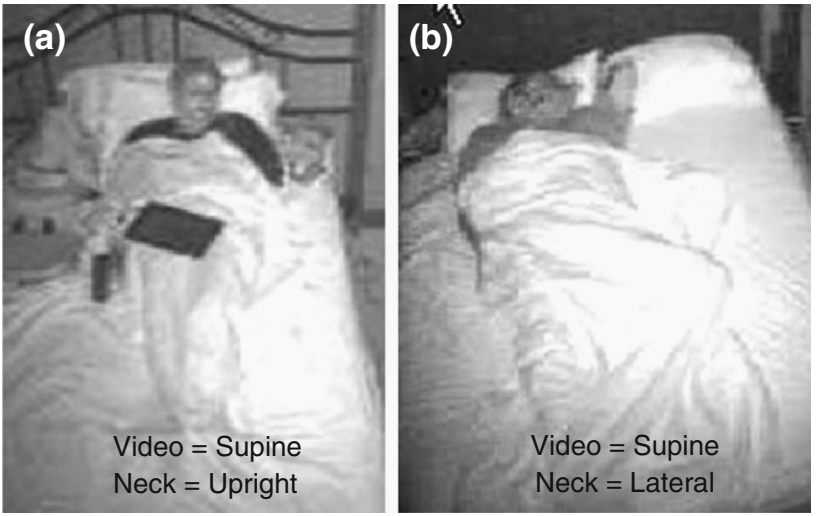

Fig. 4 Photos of two cases of gross under-reporting of supine by the neck-device with a S22 misclassified as upright by neck and supine by video, and $\mathbf{b} \mathrm{S} 12$ misclassified as lateral by neck

additional 18 min supine based on the position of the neck; subject 20 spent 15 additional minutes supine based on chest position.

During the diagnostic studies, $30 \%$ of the subjects had an AHI $<5$. Significant agreements $(p<0.0001)$ were observed between the percentages of sleep time snoring $\geq 50 \mathrm{~dB}$ from the neck and the overall, supine, and non-supine AHI $(r=0.79,0.74$, and 0.83 , respectively).

For an overall $\mathrm{AHI} \geq 10$, the sensitivity and specificity of loud snoring (i.e., $50 \mathrm{~dB}$ for $\geq 10 \%$ of sleep time) were 81 and $87 \%$, respectively, with a PPV and NPV of 87 and $81 \%$ respectively. The sensitivity and NPV improved to 87 and $88 \%$, respectively, with a clinical cut-off of $\mathrm{AHI} \geq 15$. At an $\mathrm{AHI} \geq 5$, the absence of loud snoring effectively ruled out mild OSA in $92 \%$ of the cases, and the presence of loud snoring provided a $93 \%$ probability of correctly identifying those with OSA.
Loud snoring was more robust in identifying important sleep disordered breathing in the non-supine position as compared to the supine position. For a non-supine $\mathrm{AHI} \geq 10$, the sensitivity and specificity were 82 and $100 \%$, and the PPV and NPV were 100 and $90 \%$, respectively. At an $\mathrm{AHI} \geq 15$, the non-supine sensitivity and specificity were 100 and $95 \%$. In the supine position, the clinical cut-off AHI $\geq 10$ provided the optimal accuracy with a sensitivity, specificity, PPV, and NPV of 88 , 79,82 , and $85 \%$, respectively (Table 1 ).

\subsection{Study B}

Of the 24 subjects who successfully completed night 1,21 also completed night 2 and 19 completed night 3. All available data were analyzed with the exception of one subject excluded due to missing data on Night 1 baseline (forgot to turn the device on) which limited comparisons to feedback nights 2 and 3. Subjects 9 and 13 had missing data on nights 2 and/or 3 due to not turning the neck-device on. Subjects 19 and 21 turned the neck-device off on nights 2 and 3 due to complaints of repetitive buzzing. Subject 10 turned the HST off during night 3. "Appendix 2" provides line data used for the analyses.

From this community-based cohort, $33 \%$ were benign snorers, $42 \%$ had mild or moderate OSA, and $25 \%$ had severe undiagnosed OSA (i.e., overall AHI $\geq 30$ ). Significant differences were observed in the sleep time and SE measured by HST and neck-actigraphy $(p<0.05)$ (Fig. 5a). Figure 5b confirms that the reduction in SE accuracy, measured by neck actigraphy, was attributed to very severe OSA and supported the addition of a SE threshold to further characterize OSA with the neck device.

As was observed during PSG, a very strong agreement was noted between those with loud in-home snoring plus a $\mathrm{SE} \geq 25$ and HST-AHI $(r=0.76)$. When the combination 
Table 1 Accuracy of loud snoring ( $>10 \%$ of neck-based sleep time above $50 \mathrm{~dB}$ ) in detecting $\mathrm{PSG}-\mathrm{AHI} \geq 5,10$, and 15

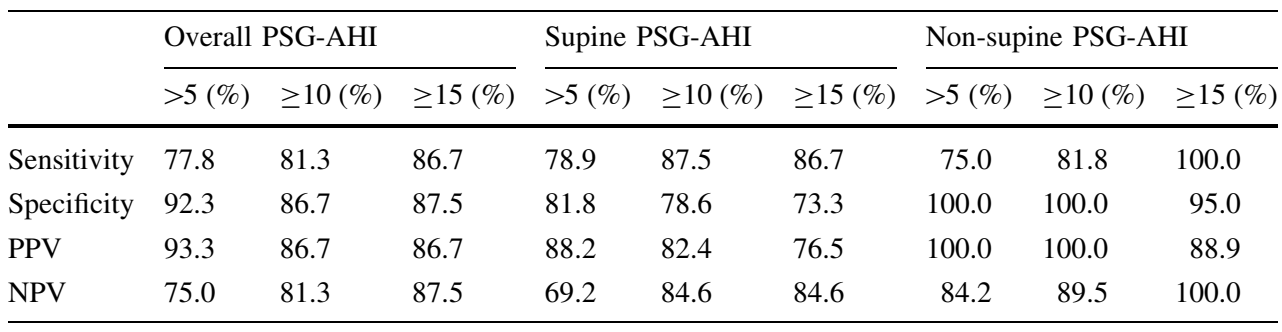

(a)

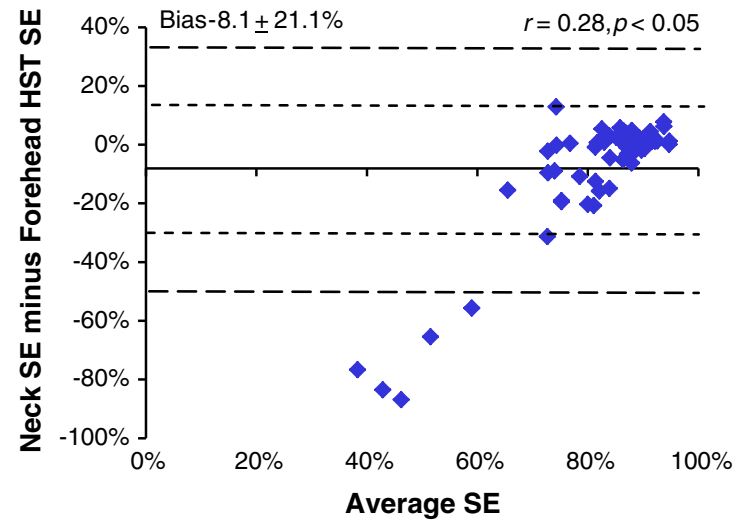

(b)

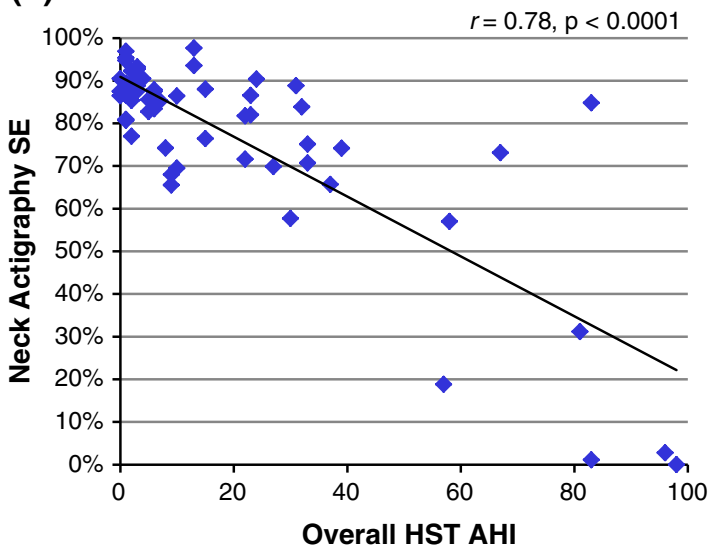

Fig. 5 a Bland-Altman plot between sleep efficiency (SE) measured by actigraphy from the neck and forehead HST across all nights, and b correlation plots between HST-AHI and sleep efficiency (SE) by neck actigraphy across all nights

Table 2 Accuracy of loud snoring and SE in detecting HST$\mathrm{AHI} \geq 5,10$, and 15

\begin{tabular}{|c|c|c|c|c|c|c|}
\hline & \multicolumn{3}{|c|}{$\begin{array}{l}\text { Night 1: HST-AHI at } \\
\text { baseline }\end{array}$} & \multicolumn{3}{|c|}{$\begin{array}{l}\text { Nights } 2 \text { and } 3 \text { : HST-AHI } \\
\text { w/positional feedback }\end{array}$} \\
\hline & $\begin{array}{l}>5 \\
(\%)\end{array}$ & $\begin{array}{l}\geq 10 \\
(\%)\end{array}$ & $\begin{array}{l}\geq 15 \\
(\%)\end{array}$ & $\begin{array}{l}>5 \\
(\%)\end{array}$ & $\begin{array}{l}\geq 10 \\
(\%)\end{array}$ & $\begin{array}{l}\geq 15 \\
(\%)\end{array}$ \\
\hline Sensitivity & 70.6 & 91.7 & 100.0 & 85.0 & 100.0 & 100.0 \\
\hline Specificity & 75.0 & 76.9 & 73.3 & 90.0 & 87.5 & 80.8 \\
\hline PPV & 85.7 & 78.6 & 71.4 & 89.5 & 84.2 & 73.7 \\
\hline NPV & 54.5 & 90.9 & 100.0 & 85.7 & 100.0 & 100.0 \\
\hline
\end{tabular}

of SE and loud snoring thresholds (i.e., SE $\leq 25$ or snoring $\geq 10 \%$ of sleep time) were compared to HST-AHI severity, the accuracies were similar to those observed during PSG. Specificities were slightly inferior at baseline, and superior on nights 2 and 3 when subjects spent the majority of time in the non-supine position. For an overall $\mathrm{AHI} \geq 10$ at baseline, the sensitivity and specificity of loud snoring were 92 and $77 \%$, respectively, with a PPV and NPV of 79 and $91 \%$ respectively. For an AHI $\geq 15$, the sensitivity and NPV improved to 100 and $100 \%$, respectively, while the specificity and PPV decreased slightly to 73 and $71 \%$, respectively. On nights 2 and 3, the sensitivity, specificity, PPV and NPV for an AHI $\geq 10$ were $100,88,84$, and $100 \%$, respectively (Table 2 ).
Sixty-nine percent of subjects with an AHI $\geq 5$ had POSA at baseline based on forehead position. The association between the percentage of sleep time supine reported from the neck and forehead was moderate $(r=0.38)$ with substantial variability (mean of differences $3 \pm 26.7 \%$ ). Feedback significantly reduced the percentage of time supine on nights 2 and 3 based on neck position $(F=13.75, p<0.0001)$ but not by forehead position.

\section{Discussion}

The purpose of this study was to evaluate the capabilities of a neck-device which uses actigraphy to measure sleep/wake and sleep position, and an acoustic microphone to record loud snoring. Studies conducted during PSG provide the gold-standard comparisons for the initial validation. Inclusion of studies during CPAP titration provides a second condition for assessment of sleep/wake, position, and snoring in over half the cases. Results were then cross-validated in-home with a community-based cohort of snorers across multiple nights with and without supine avoidance feedback.

To our knowledge, this is the first report on the use of neck actigraphy to detect sleep/wake. Typically, actigraphy accuracy is challenged by the over-estimation of sleep due to long periods of inactivity. In OSA patients, actigraphy accuracy is 
challenged by correct identification of sleep fragmentation and movement resulting from sleep-disordered breathing. For the 31 comparisons, absolute SE errors exceeded 10 in $39 \%$ of the records. By way of comparison, wrist-actigraphy based Actiwatch-64 and Fitbit reported measurement errors of 50 and $58 \%$, respectively, in 24 healthy subjects [41]. For those with an AHI $<5$, the neck-device was equivalent in accuracy to Actiwatch and superior to Fitbit in the over-reporting of sleep (i.e., bias: 9.9 vs. 9.3 and $14.5 \%$ respectively). The strong association between over-reporting of wake neck actigraphy and severe OSA was more apparent in the home studies. To achieve reasonable sleep/wake accuracy without the benefit of off-line processing of multiple parameters (i.e., actigraphy, snoring, and airflow), the wake threshold for the neck-device was set to twice the actigraphy threshold selected for forehead HST. One limitation of this study was that many conventional measures, such as sleep time, sleep latency, and wake after sleep onset, were not reported due to the number of split-night studies. A more rigorous validation of neck-actigraphy conducted during PSG studies with sleep times exceeding $5 \mathrm{~h}$ will be reported elsewhere.

A second objective of this study was to compare sleeping positions derived from transducers placed in different locations (e.g., chest, neck and head). During PSG, a substantially greater number of gross supine position detection errors were observed for both chest and neck as compared to Bignold [42], with both methods tending to over-report the supine position. Measurement of supine position during CPAP titration provides one explanation for the discrepancy. Four of six gross supine position errors by neck position, and two of six gross errors by chest position, occurred while on CPAP when patients tend to direct their head/neck toward the unit and then settle into the same position for long periods of time. Sleeping in a semi-upright position confounded both the chest and neck transducers, with chest over-reporting the supine position by $284 \mathrm{~min}$ and neck under-reporting the supine position by $116 \mathrm{~min}$. Considering the impact of position detection errors within the construct of a feedback device, false-positive supine detection may be interpreted as a nuisance and could contribute to non-compliance. False-negative detection would potentially result in non-efficacious treatment. Investigation of the false negative neck device errors during CPAP titration showed the neck was in the overlap range between supine and lateral with the head and neck rotated by more than $30^{\circ}$ and the airway in a position less susceptible to collapse [43, 44]. A prospective cross-validation of the accuracy of neck-based position assessment will be reported separately.

A third objective of this study was to assess whether loud snoring could be effective in screening for important sleepdisordered breathing. We conducted this evaluation under laboratory and home-based conditions, included at least $30 \%$ of participants without sleep disordered breathing, recruited from the community, and assessed patients under treatment conditions (i.e., CPAP titration and position restriction). In previous investigations, the percentage of time snoring based on the magnitude and duration of each snore $>40 \mathrm{~dB}$, but not $>50 \mathrm{~dB}$, was a significant predictor of positional OSA and successful outcomes with oral appliance therapy [15, 37, 44, 45]. Because snoring modulates across hypopneas and terminates during apneas, cases were reported in which the percent time snoring increased with oral appliance therapy when apneas were converted to hypopneas. We hypothesize that the strong association between snoring and AHI across a wide range of OSA severities, during both PSG and HST, resulted from a new approach whereby entire 30-s epochs were assigned loud snoring when at least one snore exceeded $50 \mathrm{~dB}$ in the duration. Including only epochs classified as sleep may also have assisted in improving snoring sensitivity and specificity.

Loud snoring for more than $10 \%$ of the night was effective in identifying important sleep disordered breathing across conventional OSA clinical cut-offs and was more accurate when subjects were in the non-supine position. During nonsupine sleep, the optimal sensitivity was obtained with PSGAHI $\geq 15(100 \%)$ and optimal specificity obtained with PSG-AHI $\geq 5 \%(100 \%)$. During supine sleep, a cut-off of PSG-AHI $\geq 10$ provided the highest sensitivity $(88 \%)$, with PSG-AHI $\geq 5$ providing the best specificity $(82 \%)$. When a HST-AHI $\geq 10$ was applied to the baseline sessions, the sensitivity and specificity from loud snoring were 91 and $77 \%$, respectively. When patients were predominantly non-supine (i.e., nights 2 and 3), the accuracy improved to 100 and $88 \%$, respectively. The accuracy in screening for OSA with the neckdevice was similar to approaches which utilized a non-contact acoustic microphone [27, 33]. Unlike the non-contact snoring approaches, the neck-device can estimate positional severity.

These findings suggest that the neck-device is capable of identifying important sleep disordered breathing in most cases. When loud snoring is measured with an acoustic microphone in combination with actigraphy-based sleep time from the neck, the SE must be considered in those with very severe OSA. The addition of SE did not affect the results obtained during PSG data because all SE were above the minimum threshold. This finding may be attributed to short-duration diagnostic sleep times resulting from the obvious need to initiate CPAP therapy.

The improved AHI detection accuracy of loud snoring in the non-supine position, during both PSG and HST, suggests possible interaction between the acoustic microphone and bed-coverings. Two false-negative findings did not appear to be solely attributed to the pillow because snoring below the loud snoring threshold was observed in both the supine and non-supine positions. Conversely, a false-positive result from loud snoring did not appear to be due to CPAP mask leakage because CPAP pressure and AHI were both low. In most cases, the vibration sounds above $50 \mathrm{~dB}$ seemed to be 
effective in assessing OSA severity at baseline and under treatment conditions. Additional studies should be conducted to investigate conditions which contribute to false-positive and false-negative results. For example, it's possible that long duration apnea or hypopnea events contribute to false negative results at $\mathrm{AHI} \geq 15$. Additional profiling of snoring patterns such as excluding snores which occur during movement, may further improve the detection capability of this measure.

Given the high prevalence of loud snoring worldwide (see "Appendix 3"), and the impact of undiagnosed OSA on both co-morbidities and medical costs, the need exists for easy, inexpensive, and accurate differentiation of benign snorers from those who should undergo a diagnostic study for OSA. As a screening tool, the results obtained with the neck-device were comparable to a single-channel Apnea Link nasal pressure device [45], with both devices capable of being selfapplied and worn in the home. The neck-device provided superior sensitivity and specificity, as compared to ApneaLink during PSG, when clinical cut-offs of AHI $\geq 5$ and $\geq 10$ were applied and inferior detection accuracy when AHI $\geq 15$ was used. One weakness of the neck-device is the potential for cross talk from a snoring bed partner. Benchtests suggest that loud bed-partner snoring can exceed $40 \mathrm{~dB}$ but rarely $50 \mathrm{~dB}$. Unlike the ApneaLink, screening of apneic and non-apneic snorers with the neck-device can include the assessment of airway position by comparing overall snoring time to supine and non-supine snoring time [25, 26].

Previous reports suggest that POSA is prevalent in $53 \%$ of patients with sleep disordered breathing (i.e., AHI $\geq 5$ plus supine AHI at least two times greater than the non-supine AHI) $[48,49]$. In the Study A group, $70 \%$ had an AHI $\geq 5$ while $45 \%$ of the cohort was identified with POSA based on body position. POSA prevalence would increase to $50 \%$ and match previous reports if subjects with supine/non-supine ratios of 1.9 and 1.8 were included. The conventional definition of POSA (i.e., supine/non-supine ratio $\geq 2.0$ ) has assumed a $4 \%$ desaturation criteria [25, 48-51]. A relaxed definition of POSA may be necessary when a $3 \%$ desaturation criteria is applied. It would be expected that a greater number of non-supine events would meet the AHI criteria given oxyhemoglobin desaturation may be less severe when lung volume is somewhat larger.

The concordance between the neck-and-chest-derived percentage of time supine was much stronger than neck and forehead supine sleep times. The prevalence of POSA detected by forehead position across a range of OSA severities was similar to previous estimates of those with mild to moderate OSA [48, 49]. Excessive measurement of supine sleep may also explain why significant changes in percent time supine from the neck were observed on nights 2 and 3 but not the forehead. As a result of the discrepancies, the agreement between positional HST-AHI and loud snoring were not compared. The accurate detection of a supine airway from the forehead is difficult because the head can be in the same angle with the torso either laterally or supine. The algorithms defining the supine position of the airway from the neck are less complicated due to its more restricted rotation. Others have argued that both head and trunk positions are clinically relevant in assessment of OSA because neither exclusively define the position of the airway $[43,44]$. This study suggests that neck position may provide an alternative approach to measuring the impact of position on airway collapsibility.

One limitation of this study was the limited assessment of the benefit of supine avoidance feedback. Study B was designed as a usability study for the neck-device, not as a treatment outcome study. It was not anticipated that a quarter of the community-based snorers would have severe undiagnosed OSA and that finding supports the need for a simple, inexpensive screening tool. Proper assessment of positional feedback outcomes, such as those conducted by Van Maanen et al. [49, 50] requires inclusion criteria limited to only those with POSA. Our study with feedback did provide insight as to conditions most conducive for vibro-tactile position therapy. For example, the two subjects, who presumed the device was not working properly because it buzzed every 20-30 min, slept $100 \%$ of the time on their back at baseline. Those who sleep exclusively supine may require a longer training/adaptation period and/or non-compliance may be greater. A report of neck strap being uncomfortable was from a female with a $54.6 \mathrm{~cm}$ neck circumference. It's unclear whether she wore the strap too tight, but it also suggests that extreme adipose tissue around the neck may influence compliance. Given the neck-device was designed to measure compliance, and can be trialed with limited effort, these concerns may be manageable with improved patient selection.

\section{Conclusions}

This report provides a full description of a neck-worn device and an initial assessment of its capabilities. Results suggest that neck actigraphy is capable of estimating sleep/wake and may be useful in predicting severe OSA. Detection of the supine airway by neck actigraphy was equivalent to the measures obtained from the chest. The supine detection accuracy of the neck-device supports further investigation for use with supine-avoidance therapy. Comparison of loud snoring, acquired with a neck-based acoustic microphone, to the AHIs obtained by PSG and HST suggest sensitivities and specificities equivalent to other simple screening tools. The combination of position assessment and OSA severity holds promise as a screening tool to differentiate positional apneic and benign snorers.

Acknowledgments The authors wish to thank Gradimir Ljubibratic, Milenko Cvetinovic PhD, and Daniela Scarfeo, Zoran Matic, Chris 
Gove, and Gene Davis for their assistance in developing the Night Shift. The technicians at Complete Sleep Solutions obtained high quality PSG data and Delmer Henninger, M.D., ABSM and James Smith, RPSGT provided video editing. All authors are salaried employees of Advanced Brain Monitoring, Inc., the sponsor of this research. No incentives were provided by the sponsor for this research effort.

Conflict of interest The authors would benefit financially if the Night Shift technology were to be sold to a third party.

Open Access This article is distributed under the terms of the Creative Commons Attribution License which permits any use, distribution, and reproduction in any medium, provided the original author(s) and the source are credited.

\section{Appendix 2}

See Table 4.

\section{Appendix 1}

See Table 3.

Table 3 Detailed data by subject used in Study A

\begin{tabular}{|c|c|c|c|c|c|c|c|c|c|c|c|c|c|c|}
\hline \multirow[t]{2}{*}{ Subj. no. } & \multirow{2}{*}{$\begin{array}{l}\text { Study } \\
\text { type }\end{array}$} & \multirow{2}{*}{$\begin{array}{l}\text { Recording } \\
\text { time-min }\end{array}$} & \multicolumn{2}{|c|}{ Sleep efficiency $(\%)$} & \multicolumn{2}{|c|}{ Overall } & \multicolumn{2}{|c|}{ Supine } & \multicolumn{2}{|c|}{ Non-supine } & \multicolumn{4}{|c|}{ Supine $(\%)$} \\
\hline & & & $\begin{array}{l}\text { PSG } \\
(\%)\end{array}$ & $\begin{array}{l}\text { Neck } \\
(\%)\end{array}$ & AHI & $\begin{array}{l}\text { Snoring } \\
(\%)\end{array}$ & AHI & $\begin{array}{l}\text { Snoring } \\
(\%)\end{array}$ & AHI & $\begin{array}{l}\text { Snoring } \\
(\%)\end{array}$ & Chest & $\begin{array}{l}\text { Chest/ } \\
\text { video }\end{array}$ & Neck & $\begin{array}{l}\text { Neck/ } \\
\text { video }\end{array}$ \\
\hline $\mathrm{S} 2 *$ & Diag. & 247 & 78.5 & 74.9 & 25.7 & 66.6 & 44.1 & 78.9 & 16.6 & 58.5 & 40.9 & 40.9 & 41.1 & 40.9 \\
\hline $\mathrm{S} 2 *$ & CPAP Titr. & 205 & 80.5 & 86.3 & 27.3 & 36.0 & 44.5 & 55.5 & 0.0 & 0.8 & 67.8 & 67.8 & 68.3 & 67.8 \\
\hline S3* & Diag. & 305 & 81.8 & 69.2 & 54.3 & 56.3 & 95.6 & 61.9 & 41.6 & 53.5 & 32.0 & 32.0 & 33.9 & 32.0 \\
\hline S3 & CPAP Titr. & 210 & 93.1 & 90.2 & 2.9 & 2.6 & 4.3 & 2.6 & 1.6 & 2.7 & 47.1 & 47.1 & 47.1 & 47.1 \\
\hline S4 & Diag. & 499 & 60.9 & 91.8 & 1.9 & 3.8 & 2.3 & 4.0 & 1.3 & 1.3 & 71.0 & 69.1 & 90.6 & 69.1 \\
\hline S5* & Diag. & 210 & 84.3 & 84.8 & 12.5 & 5.0 & 34.4 & 13.3 & 1.0 & 0.0 & 48.1 & 39.3 & 38.6 & 39.3 \\
\hline S5 & CPAP Titr. & 228 & 80.9 & 85.7 & 0.7 & 2.3 & 0.5 & 2.5 & 0.8 & 1.3 & 93.4 & 67.1 & 80.7 & 67.1 \\
\hline S6 & Diag. & 239 & 75.7 & 67.2 & 56.7 & 51.2 & 56.5 & 52.1 & 57.1 & 48.9 & 66.7 & 66.7 & 66.9 & 66.7 \\
\hline S6 & CPAP Titr. & 194 & 90.5 & 84.3 & 1.7 & 2.1 & 1.8 & 1.2 & 1.5 & 5.6 & 75.6 & 75.6 & 75.6 & 75.6 \\
\hline S11 & Diag. & 406 & 40.5 & 72.0 & 0.0 & 10.1 & 0.0 & 15.0 & 0.0 & 5.4 & 37.7 & 45.1 & 48.4 & 45.1 \\
\hline $\mathrm{S} 12$ & Diag. & 481 & 94.7 & 93.8 & 1.1 & 0.0 & 0.4 & 0.0 & 0.0 & 0.0 & 87.8 & 25.5 & 0.0 & 25.5 \\
\hline S13 & Diag. & 146 & 76.4 & 60.1 & 104.9 & 51.1 & 106.9 & 39.5 & 56.2 & 60.0 & 36.6 & 36.6 & 36.6 & 36.6 \\
\hline S13 & CPAP Titr. & 216 & 91.9 & 90.9 & 20.9 & 28.7 & 20.9 & 28.5 & N/A & N/A & 99.8 & 99.8 & 99.8 & 99.5 \\
\hline S14* & Diag. & 211 & 57.1 & 62.6 & 26.4 & 24.2 & 65.5 & 44.6 & 11.7 & 17.5 & 33.2 & 19.7 & 20.9 & 19.7 \\
\hline S14 & CPAP Titr. & 187 & 60.7 & 81.8 & 3.2 & 9.8 & 0.0 & 11.1 & 3.2 & 9.7 & 15.0 & 15.0 & 15.2 & 15.0 \\
\hline S15 & Diag. & 455 & 72.2 & 81.8 & 5.1 & 1.2 & 4.8 & 1.4 & 6.9 & 0.0 & 84.7 & 86.2 & 85.9 & 86.2 \\
\hline S16 & Diag. & 248 & 77.8 & 66.0 & 60.6 & 55.8 & 86.3 & 66.0 & 48.1 & 47.2 & 49.8 & 50.0 & 58.5 & 50.0 \\
\hline S16* & CPAP Titr. & 227 & 82.6 & 88.7 & 23.9 & 13.5 & 28.9 & 13.5 & 4.7 & N/A & 80.0 & 80.0 & 100.0 & 80.0 \\
\hline S17 & Diag. & 409 & 71.5 & 91.3 & 3.5 & 4.7 & 6.5 & 8.5 & 0.8 & 1.3 & 48.3 & 44.9 & 44.5 & 44.9 \\
\hline S18 & Diag. & 433 & 81.3 & 87.3 & 2.0 & 0.3 & 3.0 & 0.2 & 1.6 & 0.3 & 42.0 & 42.0 & 62.8 & 45.7 \\
\hline S19 & Diag. & 169 & 70.7 & 64.9 & 80.3 & 62.7 & N/A & N/A & 80.3 & 62.7 & 24.0 & 5.9 & 5.6 & 5.9 \\
\hline S19 & CPAP Titr. & 218 & 87.8 & 91.9 & 3.1 & 4.8 & 7.7 & 15.0 & 1.7 & 0.4 & 76.1 & 26.6 & 29.8 & 26.6 \\
\hline S20 & Diag. & 121 & 74.8 & 86.4 & 58.3 & 12.4 & 58.3 & 12.4 & N/A & N/A & 98.3 & 98.3 & 98.3 & 98.3 \\
\hline S20 & CPAP Titr. & 253 & 91.7 & 92.5 & 1.6 & 2.4 & 2.4 & 4.7 & 0.0 & 0.0 & 66.8 & 66.8 & 53.4 & 61.1 \\
\hline S21* & Diag. & 348 & 59.3 & 72.1 & 20.6 & 1.0 & 32.8 & 0.9 & 12.8 & 1.0 & 47.3 & 43.4 & 44.0 & 43.4 \\
\hline $\mathrm{S} 22^{*}$ & Diag. & 194 & 71.9 & 89.7 & 80.4 & 47.6 & 104.2 & 53.7 & 45.0 & 37.8 & 62.9 & 62.9 & 62.9 & 62.9 \\
\hline S22 & CPAP Titr. & 207 & 63.0 & 83.7 & 1.8 & 2.6 & 2.7 & 0.8 & 0.0 & 3.7 & 64.3 & 64.3 & 35.5 & 64.3 \\
\hline S23* & Diag. & 435 & 74.3 & 86.7 & 19.0 & 1.1 & 25.8 & 0.8 & 12.6 & 1.4 & 51.6 & 51.6 & 52.1 & 51.6 \\
\hline S24* & Diag. & 171 & 86.5 & 80.5 & 59.4 & 74.6 & 90.6 & 51.9 & 38.5 & 95.2 & 47.1 & 47.1 & 47.1 & 47.1 \\
\hline S24* & CPAP Titr. & 237 & 83.3 & 91.1 & 5.8 & 30.2 & 10.4 & 53.7 & 0.0 & 0.0 & 55.3 & 55.3 & 55.5 & 55.3 \\
\hline S25 & Diag. & 403 & 85.5 & 84.5 & 1.6 & 2.3 & 5.0 & 1.5 & 0.9 & 3.1 & 47.6 & 47.6 & 48.9 & 47.6 \\
\hline \multirow[t]{2}{*}{ POSA* } & Mean & 275 & 76.8 & 81.8 & 24.7 & 21.5 & 31.6 & 23.2 & 15.4 & 18.5 & 58.0 & 52.3 & 53.2 & 52.2 \\
\hline & SD & 109 & 12.3 & 10.0 & 29.4 & 24.2 & 35.5 & 25.0 & 22.7 & 27.1 & 21.9 & 22.8 & 26.1 & 22.6 \\
\hline
\end{tabular}

* Positional obstructive sleep apnea 


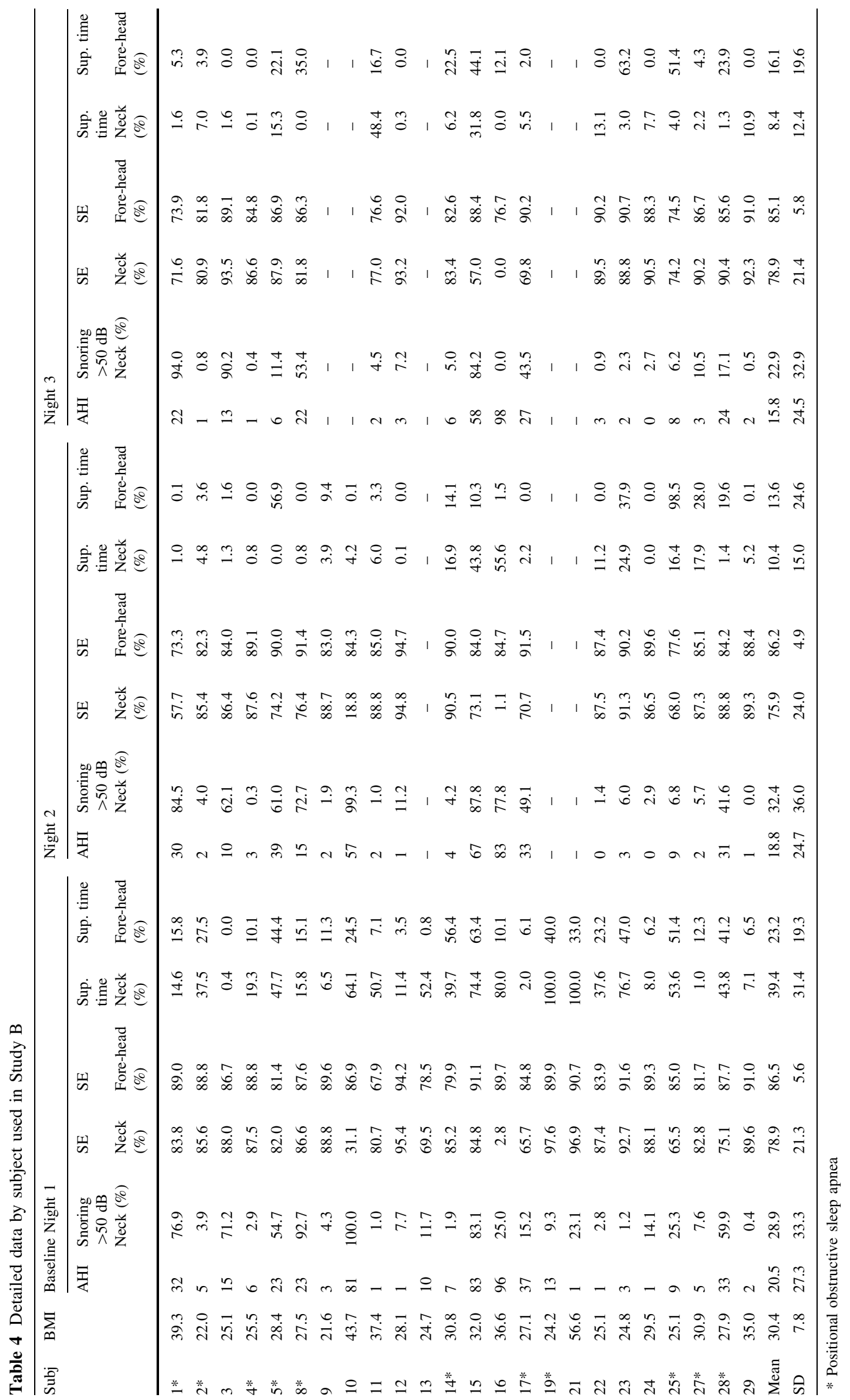




\section{Appendix 3}

See Table 5.

Table 5 Prevalence of adult habitual snorers by country and gender

\begin{tabular}{lllcll}
\hline Country & Sample size & Overall $(\%)$ & Males $(\%)$ & Females (\%) & Author \\
\hline Brazil & 3,136 & 50.5 & - & - & Noal et al. [5] \\
France & 850 & - & 34.6 & - & Teculescu et al. [6] \\
Hong Kong-students & 3,063 & 25.9 & - & - & Hui et al. [10] \\
Italian & 1,146 & 31.0 & - & - & De Benedetto et al. [7] \\
Nigeria & 370 & 31.6 & - & - & Adewole et al. [4] \\
Poland & 1,186 & 75.0 & 48.0 & 27.0 & Zielinski et al. [9] \\
Taiwan & 4,011 & 51.9 & 60.8 & 42.5 & Chuang et al. [2] \\
Turkey & 1,245 & 38.4 & 29.5 & 8.9 & Kara et al. [3] \\
United States & 5,201 & 52.0 & 33.0 & 19.0 & Enright et al. [1] \\
United Kingdom & 1,075 & 41.5 & 29.0 & 12.5 & Davey [8] \\
\hline
\end{tabular}

\section{References}

1. Enright PL, Newman AB, Wahl PM, et al. Prevalence and correlates of snoring and observed apneas in 5,201 older adults. Sleep. 1996;19(7):531-8.

2. Chuang LP, Hsu SC, Lin SW, et al. Prevalence of snoring and witnessed apnea in Taiwan adults. Chang Gung Med J. 2008;31(2):175-81.

3. Kara CO, Zencir M, Topuz B, et al. The prevalence of snoring in adult population. Kulak Burun Bogaz Ihtis Derg. 2005;14(1-2):18014.

4. Adewole OO, Ho Adeyemo, Ayeni F, et al. Prevalence and correlates of snoring among adults in Nigeria. Afr Health Sci. 2008;8(2):108-13.

5. Noal RB, Menezes AMB, Canani SF, et al. Habitual snoring and obstructive sleep apnea in adults: population-based study in Southern Brazil. Rev Saude Publica. 2008;42(2):224-33.

6. Teculescu D, Benamghar L, Hannhart B, et al. Habitual snoring: prevalence and risk factors in a sample of the French male population. Rev Mal Respir. 2007;24(3):281-7.

7. De Benedetto M, Cuda D, Leante M, et al. Snoring: an epidemiological study on 1146 adult subjects. Acta Otorhinolaryngol Ital. 1990;10(6):523-8.

8. Davey MJ. Epidemiological study of snoring from a random survey of 1,075 participants. Br Snoring Sleep Apnoea Assoc. http://www.britishsnoring.co.uk/pdf/epidem.pdf.

9. Zielinski J, Sgierska A, Polakowska M, et al. Snoring and excessive daytime somnolence among Polish middle-aged adults. Eur Respir J. 1999;14:946-50.

10. Hui DSC, Chan JKW, Ho ASS, et al. Prevalence of snoring and sleep-disordered breathing in a student population. Chest. 1999;116:1530-6.

11. Frederick IO, Qiu C, Sorensen TK, et al. The prevalence and correlates of habitual snoring during pregnancy. Sleep Breath. 2013;17(2):541-7.

12. Williams MA, Gelaye B, Qiu C, et al. Habitual snoring and asthma comorbidity among pregnancy women. J Asthma. 2011;48(1):91-7.

13. O'Brien LM, Owusu JT, Swanson LM. Habitual snoring and depressive symptoms during pregnancy. PMC Pregnancy Childbirth. 2013;13:113.
14. Puapornpong P, Neruntarat C, Manolerdthewan W. The prevalence of snoring in Thai pregnant women. J Med Assoc Thai. 2010;93:S102-5.

15. Chung JW, Enciso R, Levendowski DJ, et al. Patients with positional versus non-positional obstructive sleep apnea: a retrospective study of risk factors associated with apnea-hypopnea severity. Oral Surg Oral Med Oral Pathol Oral Radiol Endod. 2010;110:605-10.

16. Rice TB, Strollo PJ. A nuisance or nemesis: the adverse effects of snoring. Sleep. 2011;34(6):693-4.

17. Gislason T, Almqvist M, Eriksson G, et al. Prevalence of sleep apnea syndrome among Swedish men: an epidemiological study. J Clin Epidemiol. 1988;41:571-6.

18. Kripke DF, Ancoli-Istael S, Klauber MR, et al. Prevalence of sleep-disordered breathing in ages 40-64 years: a populationbased survey. Sleep. 1997;20:65-76.

19. Young T, Palta M, Dempsey J, et al. The occurrence of sleepdisordered breathing among middle-aged adults. N Engl J Med. 1993;328:1230-5.

20. Young T, Peppard PE, Gottlieb DJ. Epidemiology of obstructive sleep apnea: a population health prospective. Am J Respir Crit Care Med. 2002;165:1217-39.

21. Cho JG, Witting PK, Verma M, et al. Tissue vibration induces carotid artery endothelial dysfunction: a mechanism linking snoring and carotid atherosclerosis? Sleep. 2001;34(6):751-7.

22. Lee SA, Amis TC, Byth K, et al. Heavy snoring as a cause of carotid artery atherosclerosis. Sleep. 2008;31:1207-13.

23. Drager LF, Lorenzi-Filho G. Heavy snoring and carotid atherosclerosis: is there more than an association? Sleep. 2008;31:1335.

24. Li Y, Liu J, Wang W, et al. Association of self-reported snoring with carotid artery intima-media thickness and plaque. J Sleep Res. 2012;21:87-93.

25. Benoist LB, Morong S, van Maanen JP, et al. Evaluation of position dependency in non-apneic snorers. Eur Arch Otohinolaryngol. 2013;271(1):189-94.

26. Nakano H, Ideda T, Hayashi M, et al. Effects of body position on snoring in apneic and nonapneic snorers. Sleep. 2003;26(2):169-72.

27. Ben-Israel N, Tarasiuk A, Zigel Y. Obstructive apnea hypopnea index estimation by analysis of nocturnal snoring signals in adults. Sleep. 2012;35(9):1299-305. 
28. Fiz JA, Jane R, Sola-Soler J, et al. Continuous analysis and monitoring of snores and their relationship to the apnea-hypopnea index. Laryngoscopy. 2010;120:854-62.

29. $\mathrm{Ng} \mathrm{AK}$, Koh TS, Baey E, et al. Could formant frequencies of snore signals be an alternative means for the diagnosis of obstructive sleep apnea? Sleep Med. 2008;9:894-8.

30. Mikami T, Kojima Y, Yamamoto $M$ et al. Automatic classification of oral/nasal snoring sounds based on the acoustic properties. IEEE international conference on acoustics, speech and signal processing 2012; 609-612.

31. Emoto T, Abeyratne UR, Akutagawa M, et al. High frequency region of the snore spectra carry important information on the disease of sleep apnoea. J Med Eng Tech. 2011;35(8):425-31.

32. Pevernagie D, Aarts RM, De Meyer M. The acoustics of snoring. Sleep Med Rev. 2010;14:131-44.

33. Abeyratne UR, de Silva S, Hukins C, et al. Obstructive sleep apnea screening by integrating score feature classes. Physiol Meas. 2013;34(2):99-121.

34. The AASM manual for scoring of sleep and associated events: rules, terminology and technical specifications, 2007.

35. Chung F, Yegneswaran B, Liao P, et al. STOP questionnaire: a tool to screen patients for obstructive sleep apnea. Anesthesiology. 2008;108(5):812-21.

36. Levendowski D, Olmstead R, Popovic D, et al. Assessment of obstructive sleep apnea risk and severity in truck drivers: validation of a screening questionnaire. Sleep Diagn Ther. 2007;2(2):20-6.

37. Levendowski DJ, Morgan T, Montague J, et al. Prevalence of probable obstructive sleep apnea risk and severity in a population of dental patients. Sleep Breath. 2008;12(4):303-9.

38. Ayappa I, Norman R, Seelall V, et al. Validation of a self-applied unattended monitor for sleep disordered breathing. J Clin Sleep Med. 2008;4(1):26-37.

39. Levendowski D, Steward D, Woodson BT, et al. The impact of obstructive sleep apnea variability measured in-lab versus inhome on sample size calculations. Intl Arch Med. 2009;2:2.

40. Westbrook PR, Levendowski DJ, Cvetinovic M, et al. Description and validation of the apnea risk evaluation system: a novel method to diagnose sleep apnea-hypopnea in the home. Chest. 2005; 128:2166-75.

41. Montgomery-Downs HE, Insana SP, Bond JA. Movement toward a novel activity monitoring device. Sleep Breath. 2012;16:913-7.

42. Bignold JJ, Mercer JD, Antic NA, et al. Accurate position monitoring and improved supine-dependent obstructive sleep apnea with a new position recording and supine avoidance device. J Clin Sleep Med. 2011;7(4):376-83.

43. Van Kesteren ER, van Maanen JP, Hilgevoord AAJ, et al. Quantitative effects of trunk and head position on the apnea hypopnea index in obstructive sleep apnea. Sleep. 2011;34(8):1075-81.

44. Chung JW, Enciso R, Levendowski DJ, et al. Treatment outcomes of mandibular advancement devices in positional and nonpositional patients. Surg Oral Med Oral Pathol Oral Radiol Endod. 2010;109(5):724-31.

45. Levendowski D, Morgan T, Patrickus J, et al. In-home evaluation of efficacy and titration of a mandibular advancement device for obstructive sleep apnea. Sleep Breath. 2007;11(3):139-47.

46. Crowley KE, Rajaratnam SMW, Shea SA, et al. Evaluation of a single-channel nasal pressure device to assess obstructive sleep apnea risk in laboratory and home environments. J Clin Sleep Med. 2013;9(2):109-16.

47. Ravesloot MJL, van Maanen JP, Dun L, et al. The undervalued potential of position therapy in position-dependent snoring and obstructive sleep apnea-a review of the literature. Sleep Breath. 2013;17(1):39-49.

48. Richard W, Kox D, den Herder C, et al. The role of sleep position in obstructive sleep apnea. Eur Arch Otorhinolaryngol. 2006;263:946-50.

49. van Maanen JP, Meester KA, Dun LN, et al. The sleep position trainer: a new treatment for positional obstructive sleep apnoea. Sleep Breath. 2013;17(2):771-9.

50. van Maanen JP, Richard W, van Kesteren ER, et al. Evaluation of a new simple treatment for positional sleep apnoea patients. J Sleep Res. 2011;21(3):322-9.

51. Marklund M, Persson M, Franklin KA. Treatment success with an mandibular advancement device is related to supine-dependent sleep apnea. Chest. 2004;114:1630-5. 